\title{
Widening of the QRS Complex During Atrial Pacing: What is the Mechanism?
}

\author{
FRED MORADY, M.D. \\ From the Division of Cardiology, Department of Internal Medicine, University of Michigan, Ann Arbor, Michigan
}

\section{Case Presentation}

An electrophysiologic procedure was performed in a 50 -year-old man with a 20-year history of paroxysmal tachycardia. During sinus rhythm, the atrial-His $(\mathrm{AH})$ interval was $70 \mathrm{msec}$, the His-ventricular (HV) interval was 45 $\mathrm{msec}$, and the QRS width was $80 \mathrm{msec}$. During atrial pacing at a cycle length of $290 \mathrm{msec}$, there was widening of the QRS complex (Fig. 1). The transition from a narrow to wide QRS complex is shown at a more rapid paper speed in Figure 2. Upon cessation of pacing, sinus rhythm was present. What accounts for widening of the QRS complex during atrial pacing?

\section{Commentary}

There are three possible explanations for widening of the QRS complex during atrial pacing: induction of ventricular tachycardia, rate-related bundle branch block, and ventricular preexcitation. In this case, the ventricular

J Cardiovasc Electrophysiol, Vol. 13, pp. 627-628, June 2002.

This manuscript was processed by a guest editor.

Address for correspondence: Fred Morady, M.D., Division of Cardiology, B1F245, Department of Internal Medicine, University of Michigan, 1500 E. Medical Center Drive, Ann Arbor, MI 48109-0022. Fax: 734-936-7026; E-mail: fmorady@umich.edu cycle length remained the same as the atrial pacing cycle length when the QRS complexes became wide, and sinus rhythm was present upon cessation of atrial pacing, ruling out the induction of ventricular tachycardia. A raterelated bundle branch block is easily dismissed by the fact that there is not a His-bundle depolarization in front of the wide QRS complexes. As the QRS complexes transition from narrow to wide, the HV interval shortens from 45 to $0 \mathrm{msec}$, consistent with ventricular preexcitation.

The key to determining the mechanism of ventricular preexcitation is the location and activation sequence of the His-bundle potential. When the QRS complex is narrow, the His bundle is activated in a proximal-to-distal sequence, as would be expected when there is anterograde conduction through the His bundle. But when the QRS complex widens, activation of the His bundle reverses to a distal-to-proximal sequence, as seen in Figure 3. This is not consistent with ventricular preexcitation through an accessory AV connection. When there is fusion of the QRS complex due to anterograde conduction through both an accessory AV connection and the AV node-His-Purkinje system, the His bundle is activated in a proximal-to-distal fashion. If activation of the ventricle occurs only through the accessory $\mathrm{AV}$ connection, as is the case during antidromic reciprocating tachycardia, the activation sequence in the His bundle does reverse its direction, but His-bundle activation is de-
Figure 1. Atrial pacing at a cycle length of $290 \mathrm{msec}$. Shown are leads I, II, III, and $V_{5}$, the high right atrial electrogram (HRA), the proximal, mid, and distal Hisbundle electrograms ( $H B E p, H B E m, H B E d$ ), the right ventricular electrogram (RVA), and a stimulus channel. Paper speed is $100 \mathrm{~mm} / \mathrm{sec}$.

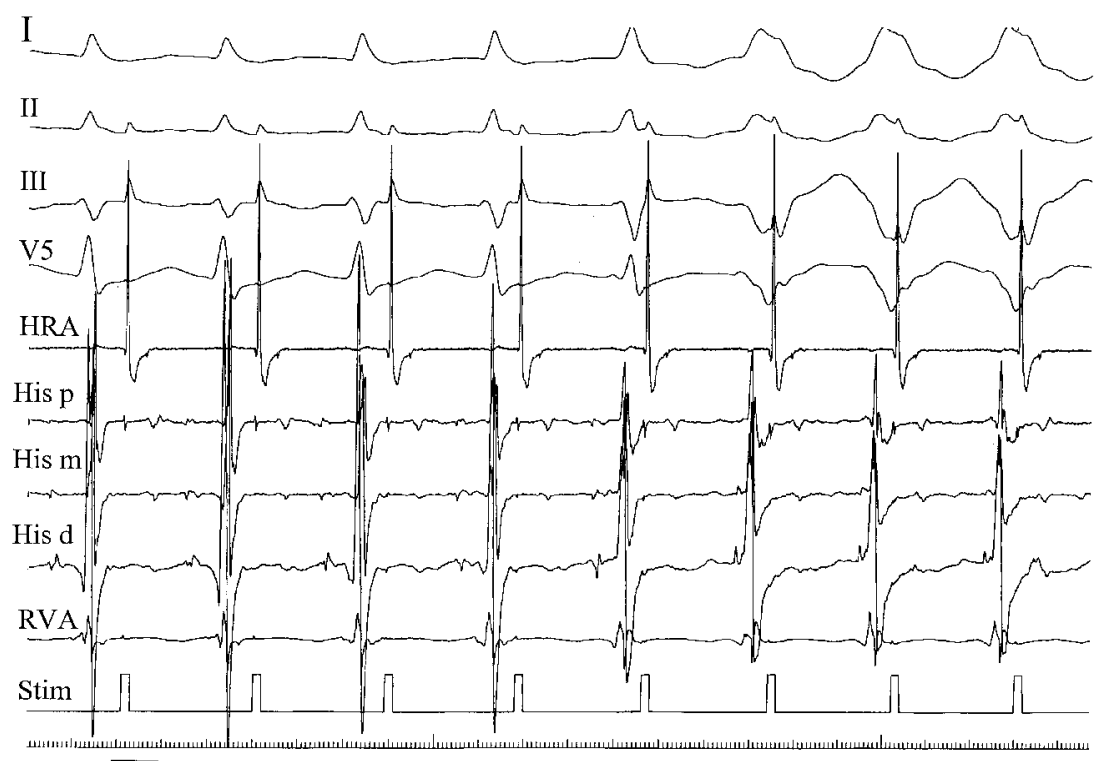

$10 \overline{\mathrm{msec}}$ 


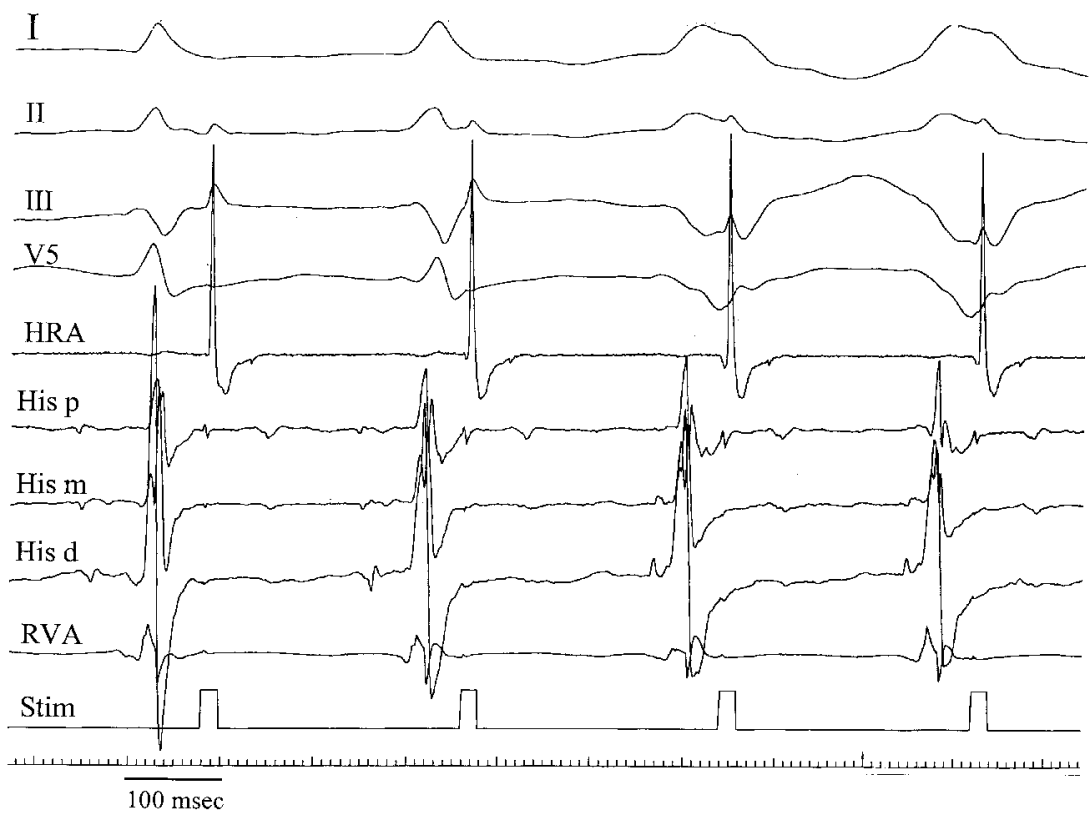

Figure 2. Transition from narrow to wide $Q R S$ complex during atrial pacing, displayed at a paper speed of $200 \mathrm{~mm} / \mathrm{sec}$. Same format as in Figure 1. layed relative to ventricular activation, because the depolarization wavefront must travel from the point of ventricular insertion of the accessory pathway to the His bundle. Therefore, an HV interval of zero in association with retrograde His-bundle activation is not compatible with ventricular preexcitation through a typical accessory pathway. Widening of the QRS complex in this case was due to anterograde conduction through an atriofascicular bypass tract. Because the bypass tract inserts into the right bundle, anterograde conduction to the ventricle and retrograde conduction to the His bundle occur simultaneously, resulting in an $\mathrm{HV}$ interval of zero. If the wavefront resulting from anterograde conduction through the atriofascicular bypass tract reaches the His bundle before the anterograde wavefront traveling through the AV node, the His bundle will be activated in retrograde fashion. In the present case, Hisbundle activation reversed when sufficient slowing in AV nodal conduction was induced by atrial pacing. The same phenomenon would occur with a nodofascicular bypass tract.

During isoproterenol infusion, an antidromic atriofascicular tachycardia was inducible. An atriofascicular potential was identified at the posterolateral tricuspid annulus during sinus rhythm, and delivery of radiofrequency energy at this site resulted in elimination of conduction through the atriofascicular bypass tract.

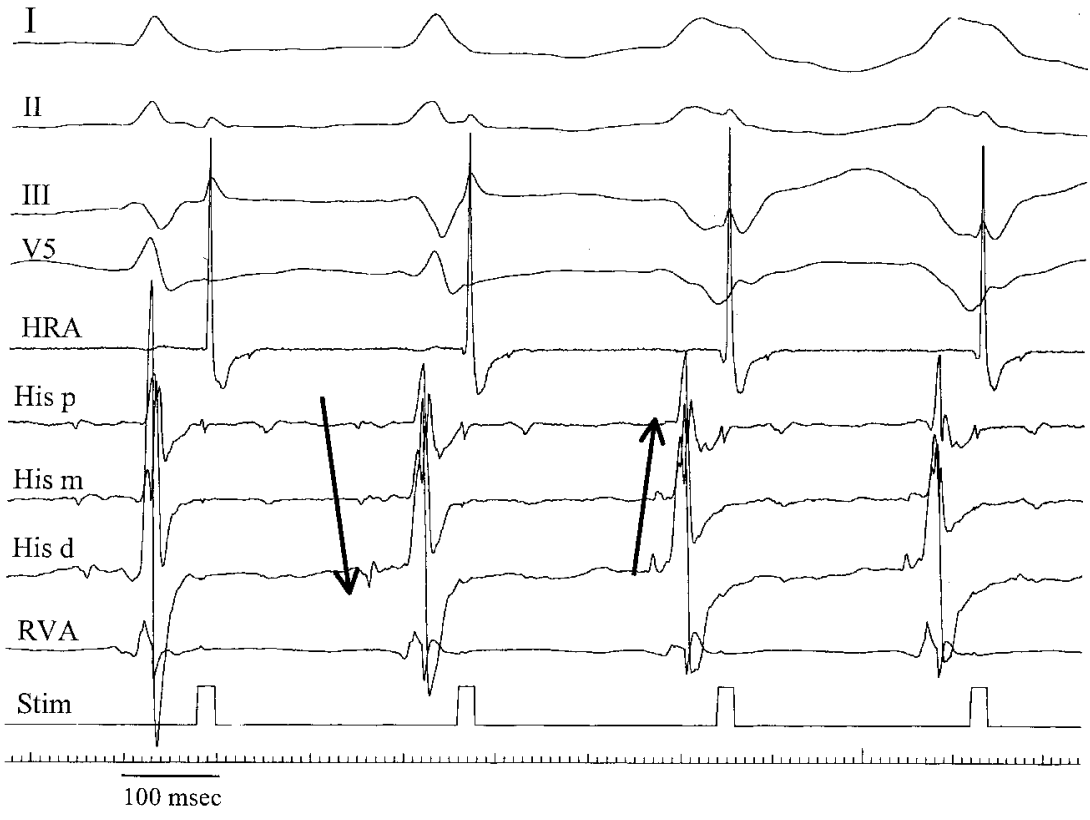

Figure 3. Same recordings as shown in Figure 2. The arrows depict the anterograde His-bundle activation that occurred when the QRS complex was narrow and the retrograde His-bundle activation that occurred when the QRS complex became wide. 